should be encouraged. In the mean time dentists should follow all the recommended measures of asepsis, disinfection, and sterilisation beginning with, among others, handwashing and care of hands and decontamination of instruments and environmental surfaces and ending with disposal of waste materials. ${ }^{3}$

Our findings are further evidence of widespread contamination by $\mathrm{HBsAg}$ in areas where carriage rates are high and reinforce the view that vaccination against hepatitis B should be extended to everyone living in such an area. ${ }^{1}$ Such a mass vaccination programme would be prohibitively expensive, however, and we therefore suggest that certainly all children aged 3 months to 12 years should be vaccinated using the cheaper but equally effective protocol developed by $u^{45}$ and accepted by both the French and Italian health authorities.

1 Piazza M. Epatite virale acuta e cronica. 4th ed. Milan: Ghedini Publ, 1986.

2 Bond WW, Petersen NJ, Favero MS. Viral hepatitis B: aspects of environmental control. Health Laboratory Science 1977;14:235-52.

3 Dental Disease Prevention Activity Group. Recommended infection-control practices for dentistry. MMWR 1986; 35:237-42.

4 Piazza M, Picciotto L, Villari R, et al. Hepatitis B immunisation with a reduced number of doses in newborn babies and children. Lancet 1985; $949-51$.

5 Piazza M, Picciotto L, Villari R, et al. Two-dose hepatitis B immunisation regimen for infants. Lancet 1985; ii:1120-1.

(Accepted l May 1987)

Clinica delle Malattie Infettive, II Facoltà di Medicina, Università di Napoli, Italy

MARCELLO PIAZZA, MD, professor and chairman

VINCENZO GUADAGNINO, MD, senior assistant

LUDOVICO PICCIOTTO, MD, senior assistant

GUGLIELMO BORGIA, MD, senior assistant

SALVATORE NAPPA, $M D$, assistant

Correspondence to: Professor M Piazza, Clinica delle Malattie Infettive, II Facoltà di Medicina, via S Pansini, 5, I-80131 Napoli, Italy.

\section{Emergency phlebography service: is it worth while?}

Many patients present with suspected deep vein thromboses, and immediate confirmation of this diagnosis allows treatment to be started and patients to be discharged earlier, If the result of investigation is normal the patient can be sent home from the casualty department. For this reason an emergency phlebography service has been established at this hospital, but the radiologists thought that too many normal phlebograms were being obtained. We undertook a prospective study to assess the value of the service.

\section{Patients, methods, and results}

For seven months all patients referred for phlebography of the leg for suspected deep vein thrombosis were assessed by a radiologist for predisposing factors and physical signs of thrombosis. The findings and provisional diagnosis of the

Deep vein thrombosis: radiologist's opinion and result of phlebography

\begin{tabular}{lcc}
\hline & \multicolumn{2}{c}{ Radiologist's opinion } \\
\cline { 2 - 3 } Result of phlebography & Normal & Thrombosis \\
\hline Normal & 48 & 17 \\
Thrombosis & 5 & 50 \\
\hline
\end{tabular}

radiologists and the results of phlebography were recorded. All phlebograms were obtained by injecting iopamidol into the foot. Altogether 120 patients ( 78 women, 42 men) were investigated; the age range was $17-90,46$ patients being over the age of 65 . Normal phlebograms were reported in 65 patients; 12 of these were inpatients and 53 were referred from the casualty department and were subsequently discharged. The table summarises the results.

\section{Comment}

Confirmation of thrombosis is necessary before starting oral anticoagulation and the most cost effective way of doing this is to use phlebography.' Sixty five of the 120 patients with deep vein thromboses suspected on clinical grounds had normal phlebograms; this correlates well with other published results. ${ }^{12}$ This does not bear out the impression that too many normal phlebograms were being obtained. Phlebography done before admission saved 53 patients a minimum of one night connected to a heparin infusion in hospital, which is equivalent to 91 patients a year.

If an emergency phlebography service is to be recommended for all hospitals how may the expected workload be reduced? All patients having phlebography should be candidates for oral anticoagulation; those who are not should not have this investigation. Patients over 65 taking oral anticoagulants have an increased incidence of haemorrhage, ${ }^{3}$ about two thirds of cases occurring secondary to ulcers and cancers etc, which increase in prevalence with age. By the age of 90 anticoagulation carries a high risk of haemorrhage and phlebography and anticoagulation would seem to be overaggressive treatment. The question, however, is where to draw the line. If patients older than 65 were treated conservatively at our hospital 78 patients would be spared phlebography each year and 30 of them admission to hospital for anticoagulation. Some of these, however, would develop pulmonary embolism. In practice the age limit is therefore likely to be higher and the savings in workload and cost less.

We believe, however, that what is needed is a non-invasive screening test for deep vein thrombosis, and one such is liquid crystal thermography. It gives immediate results with a sensitivity of $97 \%$ and a negative predictive value of $96.5 \% .^{2}$ Although the cost of the system may seem high, if used for all patients in whom deep vein thrombosis is suspected it would save 68 phlebograms a year in our hospital; the minimum cost of materials for a phlebogram is $£ 20$ so the equipment would pay for itself in three years.

This study shows that an emergency phlebography service saves unnecessary admissions and allows treatment to be started earlier for those who need it. It allows doctors to institute a plan of management confidently and frees hospital beds. The number of emergency phlebograms could be reduced by not obtaining them for those older patients in whom there are strong contraindications to anticoagulation and by using liquid crystal thermography.

We are grateful to the district research committee for a grant for secretarial help and to our colleagues for their cooperation.

1 Hull R, Hirsh J, Sackett DL, Stoddart G. Cost effectiveness of clinical diagnosis, venography, and noninvasive testing in patients with symptomatic deep-vein thrombosis. $N$ Engl f Med noninvasive testing

2 Sandler DA, Martin JF. Liquid crystal thermography as a screening test for deep-vein thrombosis. Lancet 1985;i:665-8.

3 Roos J, van Roost HE. The cause of bleeding during anticoagulant treatment. Acta Med Scand 1965;178:129-31.

(Accepted 3 fune 1987)

Department of Radiology, John Radcliffe Hospital, Oxford OX3 9DU

M J CHARIG, BSC, MRCP, senior registrar

E W L FLETCHER, MA, FRCR, consultant radiologist

Correspondence to: Dr Charig.

\section{Reversible bone loss in anorexia nervosa}

Patients with anorexia nervosa develop osteopenia, which may result in pathological fracture, including vertebral collapse. ${ }^{12}$ Susceptibility to bone fracture is a well recognised complication of premenopausal oestrogen deficiency and may occur after oophorectomy, hyperprolactinaemicamenorrhoeic syndrome, and exercise related amenorrhoea.$^{3.5}$ It has been uncertain whether it is oestrogen deficiency or the direct consequence of malnutrition that causes osteopenia in anorexia nervosa.

\section{Patients, methods, and results}

We studied four groups of subjects: 45 patients with anorexia nervosa aged 14 54 (median 26) who had fallen to a minimum of $75 \%$ of their premorbid weight and had had amenorrhoea for over a year; a companion group of 31 normal volunteers aged 19-46 (median 26) (comparison group 1); 25 patients recovered from anorexia nervosa aged 23-52 (median 36), older at the time of study but comparable with patients with current anorexia nervosa; and data from 20 aged matched patients (age range 24-48, median 36), healthy apart from breast tenderness, studied before a therapeutic trial (comparison group 2). Bone mineral density of the lumbar spine and right hip was measured with a Nova 22a dual photon absorptiometer by using gadolinium-153. The non-dominant radius was measured with a Nova GT35 single photon absorptiometer with iodine-125. 